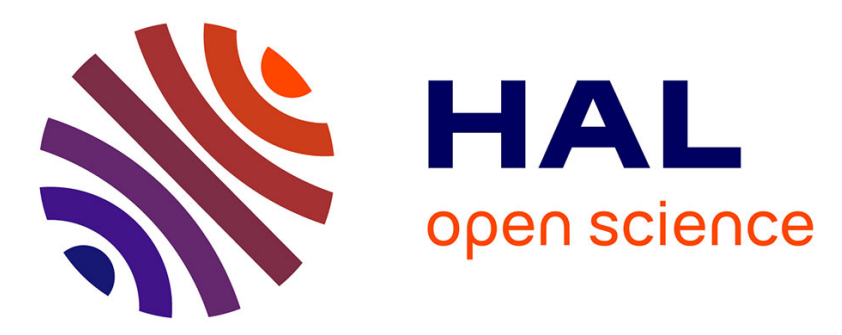

\title{
Design of a unified adaptive fuzzy observer for uncertain nonlinear systems
}

\author{
Abdesselem Boulkroune, M. Tadjine, Mohammed M'Saad, Mondher Farza
}

\section{To cite this version:}

Abdesselem Boulkroune, M. Tadjine, Mohammed M'Saad, Mondher Farza. Design of a unified adaptive fuzzy observer for uncertain nonlinear systems. Information sciences, Information Sciences, 2014, Vol. 265, pp.139-153. 10.1016/j.ins.2013.12.026 . hal-01063895

\section{HAL Id: hal-01063895 https://hal.science/hal-01063895}

Submitted on 15 Sep 2014

HAL is a multi-disciplinary open access archive for the deposit and dissemination of scientific research documents, whether they are published or not. The documents may come from teaching and research institutions in France or abroad, or from public or private research centers.
L'archive ouverte pluridisciplinaire HAL, est destinée au dépôt et à la diffusion de documents scientifiques de niveau recherche, publiés ou non, émanant des établissements d'enseignement et de recherche français ou étrangers, des laboratoires publics ou privés. 


\title{
Design of a unified adaptive fuzzy observer for uncertain nonlinear systems
}

\author{
A. Boulkroune ${ }^{\mathrm{a}, *}$, M. Tadjine $^{\mathrm{b}}$, M. M'Saad $^{\mathrm{c}}$, M. Farza $^{\mathrm{c}}$ \\ ${ }^{a}$ LAJ, Department of Automatic Control, University of Jijel, BP. 98, Ouled-Aissa, 18000 Jijel, Algeria \\ ${ }^{\mathrm{b}}$ Department of Electrical Engineering, ENP, Algiers, Algeria \\ 'GREYC, UMR 6072 CNRS, Université de Caen, ENSICAEN, 6 Bd Maréchal Juin, 14050 Caen Cedex, France
}

Keywords:

Adaptive observer

Fuzzy system

PI adaptation law

Nonlinear system

High-gain observer

Sliding-mode observer

\section{A B S T R A C T}

This paper presents an adaptive fuzzy observer for a class of uncertain nonlinear systems. More precisely, we propose a unified approach for designing such an observer with some design flexibility so that it can be easily adaptable and employed either as a high-gain or a sliding mode observer by selecting its gain appropriately. Additionally, we derive a suitable parameter adaptation law so that the proposed observer is robust with respect to ubiquitous fuzzy approximation errors and external disturbances. We also show that the observation error is ultimately bounded using a Lyapunov approach without having recourse to the usual strictly positive real (SPR) condition or a suitable observation error filtering. The effectiveness of the proposed observers is illustrated through two simulation case studies taken from the adaptive fuzzy control literature.

\section{Introduction}

In most practical situations, one is rarely if ever in the presence of a system whereby all the states variables of the system are fully measured or accessible. This particular fact has been the main motivation and driving force behind the development of the observation theory for dynamical systems for the purpose of process control and fault diagnosis. In effect, over the years there has been a considerable development in various observer design methodologies using different approaches (see list of references herein). Among these, the design of adaptive observers is quite challenging since they allow to estimate not only the state variables but the unknown system parameters as well [14] and this in the presence of modelling uncertainties.

The first nonlinear adaptive observer was proposed in [2] for single-input single-output (SISO) nonlinear systems that can be transformed into an uncertain observable canonical form. The multiple-input single-output (MISO) case has been investigated in $[22,23]$ for nonlinear systems that are linear with respect to the unknown parameters. Additionally, these systems are transformable, by using a suitable change of coordinates, into a special canonical form whereby the nonlinearities are functions of the output only. An adaptive observer with an arbitrary fast exponential convergence has been proposed in [24] for the class of MISO systems mentioned above. The multiple-input multiple-output (MIMO) case has been treated in $[5,27]$ for a particular class of Lipschitz nonlinear systems that are linear in the unknown parameters owing to an appropriate dissipative or strictly positive real (SPR) condition. In [12], an adaptive observer has been presented for a class

\footnotetext{
* Corresponding author.

E-mail addresses: boulkroune2002@yahoo.fr (A. Boulkroune), tadjine@yahoo.fr (M. Tadjine), msaad@greyc.ensicaen.fr (M. M'Saad), mfarza@greyc. ensicaen.fr (M. Farza).
} 
of time-delay systems whereby the underlying observation algorithm is designed independently of the time-delay variations, thanks to an adequate SPR condition on the observation error dynamics along with a matching condition involving the system nonlinearities.

Adaptive observers as well as adaptive controllers (see e.g. $[17,6,25,26,34,44]$ and $[18,20-21,26,30-32,35,36,38,41-43]$ ) for nonlinear systems incorporating universal approximators, namely fuzzy systems (FS) and neural networks (NN) have also received considerable attention over the past few decades. These universal approximators $[4,11,36,37]$ make it possible to relax the aforementioned structural constraints such as the linearity in the unknown parameters, the Lipschitz condition on the nonlinearity involved or the prior knowledge of the nonlinearities and the output feedback form. In [17], an adaptive observer has been proposed for SISO nonlinear systems based on the universal approximation theorem along with a suitable SPR condition on the observation error dynamics. An appropriate filtering of the observation error dynamics is used to deal with the SPR requirement. This filtering inevitably increases the order of the observer dynamics. In [29], a fuzzy adaptive observer has been developed for a class of time-delayed chaotic systems. Sufficient conditions guaranteeing robust observation performances have been established.

A set of adaptive fuzzy or neural observers has been developed for a class of uncertain nonlinear systems in $[6,25,26,34]$ without resorting to the SPR condition. In $[25,26]$, the output observation error is filtered and the state variables of the filter employed are used to design the underlying adaptation law as well as the robust compensator. The filter is mainly used to deal with the fuzzy approximation error as well as external disturbances. It is important to note that there is a kind of redundancy in these contributions due to the use of a specific estimator consisting of a chain of integrators to estimate the filter states. An adaptive observer has been developed in [6] using an appropriate filtering of the regressor vector for stability purposes. It is mostly based on the nonlinear model estimation method for automated fault diagnosis proposed in [33]. Neural adaptive observers for a large class of unknown nonlinear systems have been designed in [34] with mild assumptions without resorting to the usual SPR condition.

In this paper, we propose a unified adaptive fuzzy observer design framework for a class of uncertain nonlinear systems based on the high gain observer concept. The proposed observer presents some design flexibility in the sense that one can either obtain a high-gain observer or a sliding mode observer, depending on the choice of the output error corrective term of the latter. Also, unlike previous adaptive fuzzy or neural observers, the corrective term is nonlinear with respect to the output observation error. The main feature of the proposed observer is that, firstly, the SPR condition is no longer required and secondly, a PI parameter adaptation is used to provide a suitable robustness with respect to fuzzy approximation errors and external disturbances.

An outline of the paper is as follows: In the next section, the class of systems considered and notations employed throughout the paper are given. In Section 3, an adaptive fuzzy system is used to approximate the unknown nonlinearity involved in the system is presented. In Section 4, the adaptive fuzzy observer is derived under some given assumptions together with the underlying adaptation law. The convergence analysis of the observer is also carried out. Section 5 is devoted to simulation case studies dealing with a mass-spring-damper system and a half-car active suspension system. Finally, some conclusions are given.

\section{Notation and the class of systems considered}

Throughout the paper, $R$ denotes the set of real numbers, $R^{n}$ the set of real $n$-vectors and $R^{n \times m}$ the set of real $m \times n$ matrices. The Euclidian norm of a vector $\underline{x} \in R^{n}$ is denoted by $\|\underline{x}\|$, i.e. $\|\underline{x}\|=\sqrt{x_{1}^{2}+\cdots+x_{n}^{2}}$, and the induced norm of a matrix $A \in R^{n \times m}$, is denoted by $\|A\|_{2}$. More precisely, $\|A\|_{2}=\sqrt{\lambda_{\max }\left(A^{T} A\right)}=\sigma_{\max }(A), \lambda_{\max }(\cdot)$ and $\lambda_{\min }(\cdot)$ are largest and smallest eigenvalues of the matrix $A$ and $\sigma_{\max }(A)$ is the maximum singular value. The absolute value is denoted by $|\cdot|$.

We consider the class of $n$th order nonlinear dynamical systems of the form

$$
y^{(n)}=f\left(y, \dot{y}, \ldots, y^{(n-1)}, u\right)+d(t)
$$

or equivalently of the form

$$
\begin{aligned}
& \underline{\dot{x}}=A \underline{x}+B[f(\underline{x}, u)+d(t)] \\
& y=C \underline{x}
\end{aligned}
$$

where

$$
A=\left[\begin{array}{ccccc}
0 & 1 & 0 & \cdots & 0 \\
0 & 0 & 1 & \cdots & 0 \\
\cdots & \ldots & \ldots & \ldots & \ldots \\
0 & 0 & 0 & \ldots & 1 \\
0 & 0 & 0 & \cdots & 0
\end{array}\right], \quad B=\left[\begin{array}{c}
0 \\
0 \\
\vdots \\
0 \\
1
\end{array}\right], \quad C^{T}=\left[\begin{array}{c}
1 \\
0 \\
\vdots \\
0 \\
0
\end{array}\right]
$$

$u \in \Omega_{u} \subset R$ is the input, $y \in R$ is the output, $f$ is an unknown but continuous function, $d$ is the external bounded disturbances, i.e. $|d(t)| \leqslant D$ and $\underline{x}=\left[y, \dot{y}, \ldots, y^{(n-1)}\right]^{T}=\left[x_{1}, x_{2}, \ldots, x_{n}\right]^{T} \in \Omega_{\underline{x}} \subset R^{n}$ is the unknown state vector. 
The main objective of this paper is to develop a unified adaptive fuzzy observer design framework for the above class of system. For this, an adaptive fuzzy system is used to approximate the unknown function $f(\underline{x}, u)$.

\section{Description of the fuzzy logic system}

The basic configuration of a fuzzy logic system consists in a fuzzifier, some fuzzy IF-THEN rules, a fuzzy inference engine and a defuzzifier, as shown in Fig. 1. The fuzzy inference engine uses the fuzzy IF-THEN rules to perform a mapping from an input vector $\underline{\eta}^{T}=\left[\eta_{1}, \eta_{2}, \ldots, \eta_{p}\right] \in R^{p}$ to an output $\hat{f} \in R$. The $i$ th fuzzy rule is written as

$$
R^{(i)}: \text { if } \eta_{1} \text { is } A_{1}^{i} \text { and ... and } \eta_{p} \text { is } A_{p}^{i} \text { then } \hat{f} \text { is } f^{i}
$$

where $A_{1}^{i}, A_{2}^{i}, \ldots$, and $A_{p}^{i}$ are fuzzy variables and $f^{i}$ is the fuzzy singleton for the output in the $i$ th rule. By using the singleton fuzzifier, product inference, and center average defuzzifier, the output of the fuzzy system can be expressed as follows:

$$
\hat{f}(\underline{\eta})=\frac{\sum_{i=1}^{r} f^{i}\left(\prod_{j=1}^{p} \mu_{A_{j}}\left(\eta_{j}\right)\right)}{\sum_{i=1}^{r}\left(\prod_{j=1}^{p} \mu_{A_{j}^{i}}\left(\eta_{j}\right)\right)}=\theta^{T} \psi(\underline{\eta})
$$

where $\mu_{A_{j}^{i}}\left(\eta_{j}\right)$ is the membership function value of the fuzzy variable $\eta_{j}, r$ is the number of fuzzy rules, $\theta^{T}=\left[f^{1}, f^{2}, \ldots, f^{f}\right]$ is the adjustable parameter vector (composed of consequent parameters), and $\psi^{T}=\left[\psi^{1} \psi^{2} \cdots \psi^{r}\right]$ with

$$
\psi^{i}(\underline{\eta})=\frac{\left(\prod_{j=1}^{p} \mu_{A_{j}^{i}}\left(\eta_{j}\right)\right)}{\sum_{i=1}^{r}\left(\prod_{j=1}^{p} \mu_{A_{j}^{i}}\left(\eta_{j}\right)\right)}
$$

is the fuzzy basis function $(F B F)$. Throughout the paper, it is assumed that there always exists at least one active rule, i.e. $\sum_{i=1}^{r}\left(\prod_{j=1}^{p} \mu_{A_{j}^{i}}\left(\eta_{j}\right)\right)>0$.

It is worth noting that the fuzzy system (5) is commonly used in control applications [36,37]. Following the universal approximation results $[36,37]$, the fuzzy system (5) can approximate any nonlinear smooth function $f$ on a compact operating space to any degree of accuracy. Most importantly, it is assumed that the structure of the fuzzy system and the membership function parameters are properly specified beforehand by the designer. This means that the designer decision is needed to determine the structure of the fuzzy system, namely the pertinent inputs, the number of membership functions for each input, the membership function parameters and the number of rules. The consequent parameters, i.e. $\theta$, are determined by learning algorithms.

\section{Observer design and convergence analysis}

In this section, a unified state observer is proposed to estimate the output derivatives.

\subsection{Adaptive fuzzy observer design}

Before giving the proposed observer structure, we define some key matrices and variables. More precisely, let

1. $\Delta_{i}$ be the diagonal matrix defined by

$$
\Delta_{\lambda}=\operatorname{diag}\left[1, \frac{1}{\lambda}, \ldots, \frac{1}{\lambda^{n-1}}\right]
$$

where $\lambda>1$ is a design parameter.

2. $S$ be the unique solution of the following algebraic Lyapunov equation

$$
S+A^{T} S+S A=C^{T} C
$$

It can be shown that the solution of (8) is symmetric positive definite $[7-8,10,40]$.

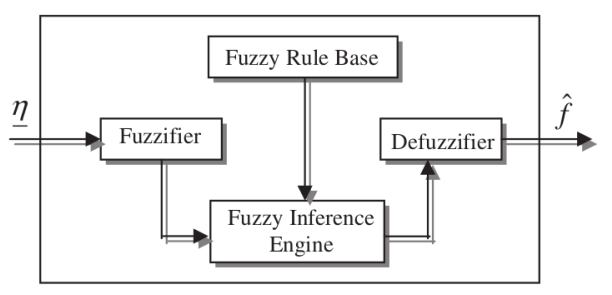

Fig. 1. The basic configuration of a fuzzy logic system. 
3. $\bar{\xi}=A_{\lambda} \underline{\xi}, \forall \underline{\xi}=\left[\xi_{1}, \xi_{2}, \ldots, \xi_{n}\right]^{T} \in R^{n}$ and $K(\underline{\xi})=\left[k_{1}\left(\xi_{1}\right), 0, \ldots, 0\right]^{T} \in R^{n}$ be a vector of smooth or non-smooth functions satisfying:

$$
\forall \underline{\xi} \in R^{n}: \bar{\xi}^{T} K\left(\xi_{1}\right) \geqslant \frac{1}{2} \bar{\xi}^{T} C^{T} C \bar{\xi}
$$

We design a unified adaptive fuzzy observer to estimate the state variables of the system (2) as follows:

$$
\begin{aligned}
& \dot{\hat{x}}=A \underline{\hat{x}}+B[\hat{f}(\underline{\hat{x}}, u, \theta)]-\lambda \Delta_{\lambda}^{-1} S^{-1} K\left(\tilde{e}_{1}\right) \\
& \hat{y}=C \underline{\hat{x}}
\end{aligned}
$$

where $\underline{\hat{x}}$ is the estimate of the state vector $\underline{x}, \hat{y}$ is the estimate of the output $y, \tilde{e}_{1}=\hat{y}-y$ is the output observation error and $\hat{f}(\underline{\hat{x}}, u, \theta)$ is the fuzzy estimate of the unknown nonlinear function $f(\underline{x}, u)$. It should be pointed out that the observer $(10)$ is composed of a copy of the system (2) together with a corrective term $\lambda \Delta_{\lambda}^{-1} S^{-1} K\left(\tilde{e}_{1}\right)$, where $K\left(\tilde{e}_{1}\right)$ is selected so that condition (9) holds.

Depending on the choice of $K\left(\tilde{e}_{1}\right)$, one can either obtain a high-gain observer or a sliding mode observer. It is worth noting that, in previous adaptive fuzzy or neural observers proposed in $[6,17,25,26,34,44]$, the corrective term is linear with respect to the output observation error, i.e. $\lambda \Delta_{\lambda}^{-1} S^{-1} K\left(\tilde{e}_{1}\right)=K_{0} \tilde{e}_{1}$.

By defining the state observation error as $\underline{\tilde{\tilde{e}}}=\underline{\hat{\hat{x}}}-\underline{\underline{x}}$, we obtain

$$
\begin{aligned}
& \underline{\dot{\tilde{\hat{e}}}}=A \underline{\tilde{e}}+B[\hat{f}(\underline{\hat{x}}, u, \theta)-f(\underline{x}, u)-d(t)]-\lambda \Delta_{\lambda}^{-1} S^{-1} K\left(\tilde{e}_{1}\right) \\
& \tilde{e}_{1}=C \underline{\tilde{e}}
\end{aligned}
$$

The unknown nonlinear function $f(\underline{x}, u)$ is approximated by a fuzzy system of the form (5) as

$$
\hat{f}(\underline{\hat{x}}, u, \theta)=\theta^{T} \psi(\underline{\hat{\eta}})
$$

where $\hat{\hat{\eta}}=\left[\underline{\hat{x}}^{T}, u\right]^{T}$ is the estimate of the vector $\underline{\eta}=\left[\underline{x}^{T}, u\right]^{T}$.

The function $f(\underline{\eta})$ can be appropriately determined according to the universal approximation theorem $[36,37]$ as follows:

$$
f(\underline{\eta})=f\left(\underline{\eta}, \theta^{*}\right)+\varepsilon(\underline{\eta})=\theta^{* T} \psi(\underline{\eta})+\varepsilon(\underline{\eta})
$$

where $\underline{\eta}=\left[\underline{x}^{T}, u\right]^{T}, \varepsilon(\underline{\eta})$ is the fuzzy approximation error and $\theta^{*}$ is the optimal parameter vector defined as follows:

$$
\theta^{*}=\underset{\theta \in \Omega_{\theta}}{\arg \min }\left[\sup _{\underline{\eta} \in \Omega_{\underline{x}} \times \Omega_{u}}\left|\theta^{T} \psi(\underline{\eta})-f(\underline{\eta})\right|\right]
$$

with $\Omega_{\theta}$ being the set of suitable bounds on $\theta$. Notice that the optimal parameter vector $\theta^{*}$ is mainly introduced for analysis purposes and its value is not needed when implementing the observer. However, the following boundedness assumption for the optimal parameter vector is customarily made [36].

Assumption 1. The optimal parameter vector satisfies

$$
\left\|\theta^{*}\right\| \leqslant M_{\theta}
$$

where $M_{\theta}$ is an unknown positive constant.

According to the universal approximation theorem [36,37] for the fuzzy logic systems, there exists a positive constant $c_{0}$ such that the following inequality holds for all $\eta \in\left(\Omega_{\underline{x}} \times \Omega_{u}\right)$ :

$$
|\varepsilon(\underline{\eta})| \leqslant c_{0}
$$

Using (12) and (13), the observation error dynamics (11) becomes

$$
\begin{aligned}
& \dot{\tilde{\tilde{e}}}=A \underline{\tilde{e}}+B\left[\tilde{\theta}^{T} \psi(\hat{\hat{\eta}})+w\right]-\lambda \Delta_{\lambda}^{-1} S^{-1} K\left(\tilde{e}_{1}\right) \\
& \tilde{e}_{1}=C \underline{\tilde{e}}
\end{aligned}
$$

where $\tilde{\theta}=\theta-\theta^{*}$ is the parameter error vector and

$$
w=\theta^{* T}[\psi(\underline{\hat{\eta}})-\psi(\underline{\eta})]-\varepsilon(\underline{\eta})-d(t)
$$

represents the lumped disturbances term induced by the state observation error, fuzzy approximation error and external disturbances.

To simplify the stability analysis, let us define a scaling transformation as follows

$$
\underline{z}=\Delta_{\lambda} \underline{\tilde{e}}
$$


One can easily show that $\underline{z}$ has the following special properties:

$$
\begin{aligned}
& \text { (i) }\|\underline{z}\| \leqslant\|\underline{\tilde{e}}\| \leqslant \lambda^{n-1}\|\underline{z}\| \\
& \text { (ii) } \quad C \underline{z}=z_{1}=C \underline{\tilde{e}}=\tilde{e}_{1}
\end{aligned}
$$

Since $\Delta_{\lambda} A \Delta_{\lambda}^{-1}=\lambda A$ and taking into account the fact that $K\left(\tilde{e}_{1}\right)=K\left(z_{1}\right)$, system (17) can be rewritten in term of $\underline{z}$ as follows:

$$
\begin{aligned}
& \underline{\dot{z}}=\lambda A \underline{z}-\lambda S^{-1} K\left(z_{1}\right)+\Delta_{\lambda} B\left[\tilde{\theta}^{T} \psi(\underline{\hat{\eta}})+w\right] \\
& z_{1}=C \underline{z}
\end{aligned}
$$

For the convergence analysis of the observer, the following lemma is required.

Lemma 1. There exists a positive constant $c_{1}$ such that

$$
|w| \leqslant c_{1}
$$

Proof of lemma 1. From (15), (16) and (18), one has

$$
|w|=\left|\theta^{* T}[\psi(\underline{\hat{\eta}})-\psi(\underline{\eta})]-\varepsilon(\underline{\eta})-d(t)\right| \leqslant\left|\theta^{* T}[\psi(\underline{\hat{\eta}})-\psi(\underline{\eta})]\right|+|\varepsilon(\underline{\eta})|+|d(t)| \leqslant M_{\theta} \sup _{t}\|\psi(\underline{\hat{\eta}})-\psi(\underline{\eta})\|+c_{0}+D \leqslant c_{1}
$$

where $c_{1}=M_{\theta} \sup _{t}\|\psi(\underline{\hat{\eta}})-\psi(\underline{\eta})\|+c_{0}+D$.

\subsection{Construction of the adaptive law}

Since $\tilde{e}_{1}$ is available for measurement, the fuzzy parameters are updated according to the following PI algorithm

$$
\dot{\theta}+\gamma_{2} \dot{\delta}=-\sigma \gamma_{1} \theta-\gamma_{1} \tilde{e}_{1} \psi(\underline{\hat{\eta}})
$$

where $\gamma_{1}, \gamma_{2}$, and $\sigma>0$ are design constants. $\delta \in R^{r}$, where $r$ is the number of the fuzzy rules, is a design function which can be selected as follows:

$$
\delta=\sigma \theta+\tilde{e}_{1} \psi(\underline{\hat{\eta}})
$$

It is worth noting that the adaptation law (25) is augmented by two terms for different purposes. The $\sigma$-modification term [13] ensures the parameter estimates boundedness, while the proportional term $\delta$ enhances the stability requirement of the adaptive fuzzy system as it will be shown later. More specifically, the proportional term permits to drop the robust compensator used in $[17,25,26]$; the latter is generally added to the adaptive fuzzy term, i.e. $\hat{f}(\underline{\hat{x}}, u, \theta)$, in order to deal with the approximation errors and external disturbances.

\subsection{Observer convergence}

The observer performances are given by the following theorem.

Theorem 1. Consider the observer (10) and let the parameter vector $\theta$ be updated by the PI adaptation law (25). Then, the observation error $\underline{\tilde{e}}$ and the parameter estimation errors $\tilde{\theta}$ are uniformly ultimately bounded (UUB). Furthermore, the observation error converges in a neighborhood of the origin.

Proof. Define the following Lyapunov function

$$
V=V_{1}+\beta V_{2}
$$

where $\beta=1 / \lambda^{2 n-2}$,

$$
V_{1}=\underline{z}^{T} S \underline{z}
$$

and,

$$
V_{2}=\frac{1}{2 \gamma_{1}}\left(\tilde{\theta}+\gamma_{2} \delta\right)^{T}\left(\tilde{\theta}+\gamma_{2} \delta\right)
$$

Differentiating $V_{1}$ along the solution (22) and using (8), one obtains

$$
\dot{V}_{1}=\underline{z}^{T} S \underline{\dot{z}}+\underline{\dot{z}}^{T} S \underline{z}=-\lambda \underline{z} \underline{z}^{T} S \underline{z}-2 \lambda\left[\underline{z}^{T} K\left(z_{1}\right)-0.5 \underline{z}^{T} C^{T} C \underline{z}\right]+2 \underline{z}^{T} S \Delta_{\lambda} B\left[\tilde{\theta}^{T} \psi(\underline{\hat{\eta}})+w\right]
$$

Due to the special forms of the matrix $\Delta_{\lambda}$ and vector $B$, it can be easily shown that $S \Delta_{\lambda} B=\sqrt{\beta} S B$. 
Now, using Lemma 1, we get

$$
\begin{aligned}
\dot{V}_{1} & \leqslant-\lambda \lambda_{\min }(S)\|\underline{z}\|^{2}-2 \lambda\left[\underline{z}^{T} K\left(z_{1}\right)-0.5 \underline{z}^{T} C^{T} C \underline{z}\right]+2 \sqrt{\beta}\left|\underline{z}^{T} S B \| \tilde{\theta}^{T} \psi(\hat{\eta})+w\right| \\
& \leqslant-\lambda \lambda_{\min }(S)\|\underline{z}\|^{2}-2 \lambda\left[\underline{z}^{T} K\left(z_{1}\right)-0.5 \underline{z}^{T} C^{T} C \underline{z}\right]+2 \sqrt{\beta} c_{2}\|S B\|\|\underline{z}\|\|\tilde{\theta}\|+2 \sqrt{\beta} c_{1}\|S B\|\|\underline{z}\| \\
& =-\lambda \lambda_{\min }(S)\|\underline{z}\|^{2}-2 \lambda\left[\underline{z}^{T} K\left(z_{1}\right)-0.5 \underline{z}^{T} C^{T} C \underline{z}\right]+2 \sqrt{\beta} c_{4}\|\underline{z}\|\|\tilde{\theta}\|+2 \sqrt{\beta} c_{3}\|\underline{z}\|
\end{aligned}
$$

where $c_{2}=\sup _{t}\|\psi(\hat{\eta})\|, c_{3}=c_{1}\|S B\|, c_{4}=c_{2}\|S B\|$ and $\lambda_{\min }(S)$ is the smallest eigenvalue of the matrix $S$.

The time-derivative of (29) along the solution of (25) is given by

$$
\dot{V}_{2}=\frac{1}{\gamma_{1}}\left(\tilde{\theta}+\gamma_{2} \delta\right)^{T}\left(\dot{\tilde{\theta}}+\gamma_{2} \dot{\delta}\right)=\left(\tilde{\theta}+\gamma_{2} \delta\right)^{T}\left(-\sigma \theta-\tilde{e}_{1} \psi(\underline{\hat{\eta}})\right)=-\sigma \tilde{\theta}^{T} \theta-\tilde{e}_{1} \tilde{\theta}^{T} \psi(\underline{\hat{\eta}})-\gamma_{2} \delta^{T}\left(\sigma \theta+\tilde{e}_{1} \psi(\underline{\hat{\eta}})\right)
$$

where $\dot{\tilde{\theta}}=\dot{\theta}-\dot{\theta}^{*}=\dot{\theta}$.

Since $2 \tilde{\theta}^{T} \theta \geqslant\|\tilde{\theta}\|^{2}-\left\|\theta^{*}\right\|^{2}$, we have

$$
\dot{V}_{2} \leqslant-\frac{\sigma}{2}\|\tilde{\theta}\|^{2}+\frac{\sigma}{2}\left\|\theta^{*}\right\|^{2}-\tilde{e}_{1} \tilde{\theta}^{T} \psi(\underline{\hat{\eta}})-\gamma_{2} \delta^{T}\left(\sigma \theta+\tilde{e}_{1} \psi(\underline{\hat{\eta}})\right) \leqslant-\frac{\sigma}{2}\|\tilde{\theta}\|^{2}+\frac{\sigma}{2}\left\|\theta^{*}\right\|^{2}+c_{2} \mid \tilde{e}_{1}\|\tilde{\theta}\|-\gamma_{2} \delta^{T}\left(\sigma \theta+\tilde{e}_{1} \psi(\underline{\hat{\eta}})\right)
$$

where $c_{2}=\sup _{t}\|\psi(\hat{\eta})\|$.

It is worth noting that the choice of $\delta$ defined by Eq. (26) allows to introduce a negative term, in the expression $\dot{V}_{2}$, which can be important provided that $\gamma_{2}$ is large enough.

From (31) and (33), the time derivative of (27) can be bounded as follows

$$
\begin{aligned}
\dot{V} \leqslant & -\lambda \lambda_{\min }(S)\|\underline{z}\|^{2}-2 \lambda\left[\underline{z}^{T} K\left(z_{1}\right)-0.5 \underline{z}^{T} C^{T} C \underline{z}\right]+2 \sqrt{\beta} c_{4}\|\underline{z}\|\|\tilde{\theta}\|+2 \sqrt{\beta} c_{3}\|\underline{z}\|-\frac{\beta \sigma}{2}\|\tilde{\theta}\|^{2}+\frac{\beta \sigma}{2}\left\|\theta^{*}\right\|^{2}+\beta c_{2} \mid \tilde{e}_{1}\|\tilde{\theta}\| \\
& -\beta \gamma_{2} \delta^{T}\left(\sigma \theta+\tilde{e}_{1} \psi(\underline{\hat{\eta}})\right)
\end{aligned}
$$

Using the following inequalities

$$
\begin{aligned}
& 2 \sqrt{\beta} c_{4}\|\underline{z}\|\|\tilde{\theta}\| \leqslant \frac{c_{4}^{2}}{\alpha_{1}}\|\underline{z}\|^{2}+\beta \alpha_{1}\|\tilde{\theta}\|^{2} \\
& 2 \sqrt{\beta} c_{3}\|\underline{z}\| \leqslant \frac{1}{\alpha_{2}}\|\underline{z}\|^{2}+\beta \alpha_{2} c_{3}^{2} \\
& \beta c_{2} \mid \tilde{e}_{1}\|\tilde{\theta}\| \leqslant \frac{\beta c_{2}^{2}}{4 \alpha_{3}}\|\tilde{e}\|^{2}+\beta \alpha_{3}\|\tilde{\theta}\|^{2} \leqslant \frac{c_{2}^{2}}{4 \alpha_{3}}\|\underline{z}\|^{2}+\beta \alpha_{3}\|\tilde{\theta}\|^{2}
\end{aligned}
$$

where $\alpha_{1}, \alpha_{2}$ and $\alpha_{3}>0$, one gets

$$
\dot{V} \leqslant-\left(\lambda \lambda_{\min }(S)-\bar{\lambda}\right)\|\underline{z}\|^{2}-2 \lambda\left[\underline{z}^{T} K\left(z_{1}\right)-0.5 \underline{z}^{T} C^{T} C \underline{z}\right]-0.5 \beta(\sigma-\bar{\sigma})\|\tilde{\theta}\|^{2}-\beta \gamma_{2} \delta^{T}\left(\sigma \theta+\tilde{e}_{1} \psi(\underline{\hat{\eta}})\right)+\beta \bar{\mu}
$$

where

$$
\bar{\lambda}=\frac{c_{4}^{2}}{\alpha_{1}}+\frac{1}{\alpha_{2}}+\frac{c_{2}^{2}}{4 \alpha_{3}}, \quad \bar{\sigma}=2\left(\alpha_{1}+\alpha_{3}\right) \quad \text { and } \quad \bar{\mu}=\frac{\sigma}{2}\left\|\theta^{*}\right\|^{2}+\alpha_{2} c_{3}^{2}
$$

If $K\left(z_{1}\right)$ and $\delta$ are designed according to (9) and (26), respectively, then the terms $\left[\underline{z}^{T} K\left(z_{1}\right)-\underline{z}^{T} C^{T} C \underline{z}\right]$ and $\gamma_{2} \delta^{T}\left(\sigma \theta+\tilde{e}_{1} \psi(\underline{\hat{\eta}})\right)$ become positive.

Using (20), we obtain

$$
\dot{V} \leqslant-\beta\left(\lambda \lambda_{\min }(S)-\bar{\lambda}\right)\|\underline{\tilde{e}}\|^{2}-0.5 \beta(\sigma-\bar{\sigma})\|\tilde{\theta}\|^{2}+\beta \bar{\mu}
$$

Choosing $\lambda>\bar{\lambda} / \lambda_{\min }(S)$ and $\sigma>\bar{\sigma}$, one can guarantee that $\dot{V}$ is negative as long as $\underline{\tilde{e}}$ is outside the compact set $\Omega_{\underline{\underline{e}}}$ defined as

$$
\Omega_{\underline{\underline{e}}}=\left\{\underline{\tilde{e}}\|\underline{\tilde{e}}\| \leqslant \sqrt{\frac{\bar{\mu}}{\lambda \lambda_{\min }(S)-\bar{\lambda}}}\right\}
$$

According to the standard Lyapunov theorem (see [14]), one concludes that $\underline{\underline{e}}$ is bounded and will converge to $\Omega_{\underline{e}}$. Moreover, the radius of $\Omega_{\underline{\underline{e}}}$ can be made arbitrary small if $\lambda$ is chosen to be sufficiently large.

The parameter error vector $\tilde{\theta}$ is also bounded and converges to $\Omega_{\tilde{\theta}}$ which is defined as follows

$$
\Omega_{\tilde{\theta}}=\left\{\tilde{\theta} \mid\|\tilde{\theta}\| \leqslant \sqrt{\frac{2 \bar{\mu}}{\sigma-\bar{\sigma}}}\right\}
$$

The boundedness of $\theta$ results from that of $\tilde{\theta}$ and Assumption 1, i.e. $\theta^{*} \in L_{\infty}$. This completes the proof of the theorem. To summarize, Fig. 2 shows the overall scheme of the unified adaptive fuzzy observer. 


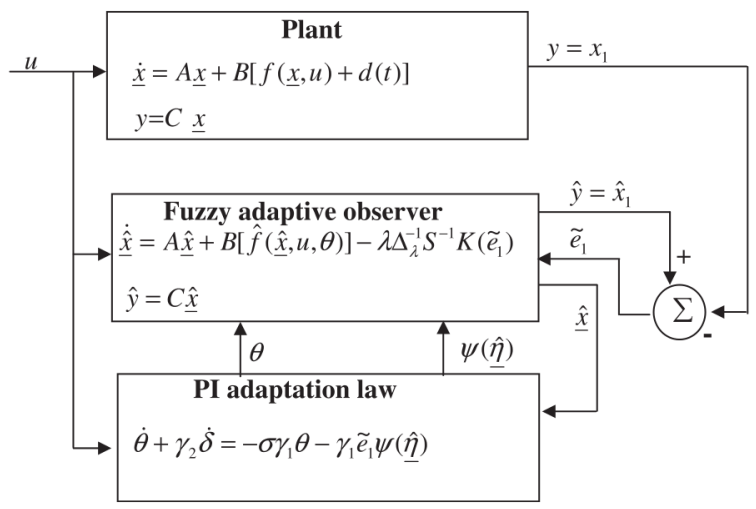

Fig. 2. Over all scheme of the unified adaptive fuzzy observer.

\subsection{Some general remarks}

Remark 1. The main characteristics of the proposed framework of the adaptive fuzzy observer design can be summarized as follows:

1. The unifying feature of the high gain concept through a suitable design function.

2. The requirements of an off-line learning phase, the persistent excitation condition and the prior knowledge of an upper bound on the unknown ideal parameters are not required at all.

3. The usual SPR condition has been dropped without resorting to any filtering unlike in $[6,25,26]$.

4. As in the universal approximator-based observation algorithms $[17,6,25,26]$, the nonlinear function $f(\underline{x}, u)$ is not assumed to be known, Lipschitz or linear in the unknown parameters.

5. The approximation error and external disturbances can be appropriately reduced in the proposed observation design framework thanks to the proportional action involved in the parameter adaptation law unlike in [17,25,26].

6. The number of design parameters is relatively small, namely $\gamma_{1}, \gamma_{2}, \sigma, \lambda$ and $k_{0}$, compared to those employed in [17,25] where seven and nine design parameters should be respectively specified.

Remark 2. The adaptive law (25) can be rewritten as follows

$$
\theta=\int_{0}^{t}\left[-\sigma \gamma_{1} \theta-\gamma_{1} \tilde{e}_{1} \psi(\underline{\hat{\eta}})\right] d \tau-\gamma_{2}\left[\sigma \theta+\tilde{e}_{1} \psi(\underline{\hat{\eta}})\right]
$$

Consequently, it cannot be used directly because of the underlying algebraic loop problem; i.e. $\theta$ appears in both the left-hand and right-hand sides of (39). To avoid this problem, one simply rewrites (39) as follows:

$$
\theta=\frac{1}{1+\gamma_{2} \sigma}\left[\int_{0}^{t}\left[-\sigma \gamma_{1} \theta-\gamma_{1} \tilde{e}_{1} \psi(\underline{\hat{\eta}})\right] d \tau-\gamma_{2} \tilde{e}_{1} \psi(\underline{\hat{\eta}})\right]
$$

\section{Remark 3.}

1. Table 1 summarizes a set of observers that can be obtained from appropriate specification of the design function $K\left(\tilde{e}_{1}\right)$. Sign, Sat, Tanh, Sinh and Arctan respectively denote the usual sign function, the usual saturation function, the hyperbolic tangent function, the hyperbolic sine function and the inverse tangent function and $k_{0}, \varepsilon$, and $p>0$ are real numbers. Notice that the name "sliding mode observer" of the observers 2-8 is inspired from the control literature [9,15,16].

2. Table 2 summarizes another set of sliding mode observers that can be obtained by exploring the involved design function. It is very easy to check that the considered functions in Table 2 satisfy the condition (9) for a relatively high value of $l$, e.g. the observer with $K_{S M}\left(\tilde{e}_{1}\right)=I C^{T}(C \operatorname{sign}(\underline{\tilde{e}}))$ satisfies the condition (9) for $l \geqslant \frac{1}{2} \sup _{t}\left|\tilde{e}_{1}\right|$. This condition on the gain $l$ is commonly used in the control literature $[1,39]$.

\section{Simulation studies}

Simulation studies are carried out to show the effectiveness of the proposed adaptive fuzzy observers. Two observation problems are considered to this end. The first one concerns a mass-spring-damper system, while the second one concerns a half-car suspension system. 
Table 1

High-gain and sliding mode observers (first type), obtained according to the choice of $K\left(\tilde{e}_{1}\right)$.

\begin{tabular}{|c|c|}
\hline$K\left(\tilde{e}_{1}\right)$ & Observer type \\
\hline (1) $K_{H G}\left(\tilde{e}_{1}\right)=C^{T}(C \underline{\tilde{e}})$ & High-gain observer [28] \\
\hline (2) $K_{S M}\left(\tilde{e}_{1}\right)=C^{T}(C \underline{\tilde{e}})+I C^{T}(C \operatorname{sign}(\underline{\tilde{e}}))$ & (Non-smooth) Sliding mode observer, $[15,16]$ \\
\hline (3) $K_{S M}\left(\tilde{e}_{1}\right)=C^{T}(C \underline{\tilde{e}})+I C^{T}\left(C \operatorname{Tanh}\left(k_{0} \underline{\tilde{e}}\right)\right)$ & (Smooth) Sliding mode observer [9] \\
\hline (4) $K_{S M}\left(\tilde{e}_{1}\right)=C^{T}(C \underline{\tilde{e}})+l C^{T}\left(C \operatorname{Sinh}\left(k_{o} \tilde{\tilde{e}}\right)\right)$ & (Smooth) Sliding mode observer \\
\hline (5) $K_{S M}\left(\tilde{e}_{1}\right)=C^{T}(C \underline{\tilde{e}})+l C^{T}\left(\operatorname{CArctan}\left(k_{0} \underline{\tilde{e}}\right)\right)$ & (Smooth) Sliding mode observer \\
\hline (6) $K_{S M}\left(\tilde{e}_{1}\right)=C^{T}(C \underline{\tilde{e}})+l C^{T}(\operatorname{CSat}(\underline{\tilde{e}}))$ & (Smooth) Sliding mode observer \\
\hline (7) $K_{S M}\left(\tilde{e}_{1}\right)=C^{T}(C \underline{\tilde{e}})+l C^{T}\left(C \underline{\tilde{e}} /\left(\varepsilon_{o}+|C \underline{\tilde{e}}|\right)\right)$ & (Smooth) Sliding mode observer \\
\hline (8) $K_{S M}\left(\tilde{e}_{1}\right)=C^{T}(C \underline{\tilde{e}})+l(|C \underline{\tilde{e}}|)^{P} C^{T} C \operatorname{sign}(\underline{\tilde{e}})$ & (Non-smooth) Sliding mode observer \\
\hline
\end{tabular}

Table 2

Sliding mode observers (second type) obtained according to the choice of $K\left(\tilde{e}_{1}\right)$.

\begin{tabular}{ll}
\hline$K\left(\tilde{e}_{1}\right)$ & Observer type \\
\hline (1) $K_{S M}\left(\tilde{e}_{1}\right)=l C^{T}(C \operatorname{sign}(\underline{\tilde{e}}))$ & (Non-smooth) Sliding mode observer [1] \\
(2) $K_{S M}\left(\tilde{e}_{1}\right)=l C^{T}\left(C \operatorname{Tanh}\left(k_{0} \underline{\tilde{e}}\right)\right)$ & (Smooth) Sliding mode observer \\
(3) $K_{S M}\left(\tilde{e}_{1}\right)=l C^{T}\left(C \operatorname{Sinh}\left(k_{0} \underline{\tilde{e}}\right)\right)$ & (Smooth) Sliding mode observer \\
(4) $K_{S M}\left(\tilde{e}_{1}\right)=l C^{T}\left(C \operatorname{Arctan}\left(k_{0} \underline{\tilde{e}}\right)\right)$ & (Smooth) Sliding mode observer \\
(5) $K_{S M}\left(\tilde{e}_{1}\right)=l C^{T}(C \operatorname{Sat}(\underline{\tilde{e}}))$ & (Smooth) Sliding mode observer \\
(6) $K_{S M}\left(\tilde{e}_{1}\right)=l C^{T}\left(C \underline{\tilde{e}} /\left(\varepsilon_{o}+|C \underline{\tilde{e}}|\right)\right)$ & (Smooth) Sliding mode observer \\
(7) $K_{S M}\left(\tilde{e}_{1}\right)=l(|C \underline{\tilde{e}}|)^{0.5} C^{T} C \operatorname{sign}(\underline{\tilde{e}})$ & (Non-smooth) Sliding mode observer [3]
\end{tabular}

Example 1. Consider the mass-spring-damper system shown in Fig. 3 whose dynamics are described by [25]:

$$
\begin{aligned}
& \dot{x}_{1}=x_{2} \\
& \dot{x}_{2}=\frac{-f_{k}(\underline{x})-f_{B}(\underline{x})+u+d}{M}
\end{aligned}
$$

where $\underline{x}=\left[x_{1}, x_{2}\right]^{T}$ is the state vector, $y=x_{1}$ represents the displacement of the mass, $x_{2}$ represents its velocity, $f_{k}(\underline{x})$ is the spring force due to spring constant $K, f_{B}(\underline{x})$ is the friction force due to friction constant $B, M$ is the body mass, $u$ is the applied force and $d$ denotes the disturbances.

In the following simulation, the nominal parameters and their corresponding uncertainties are selected as follows: $M_{0}=1 \mathrm{Kg}, K_{0}=2$ and $B_{0}=2$ and $\Delta M=0.1 \sin \left(x_{1}\right) \mathrm{kg}, \Delta K=0.5$ and $\Delta B=0.5$. Furthermore, the nonlinear spring, the friction forces and the disturbance effects are assumed to be modelled by $f_{k}(\underline{x})=K_{0} x_{1}+\Delta K x_{1}^{3}, f_{B}(\underline{x})=B_{0} x_{2}+\Delta B x_{2}^{2}$ and $d(t)=0.2 \sin (2 t) \exp (-0.1 t)$.

Solving the matrix Eq. (8), one gets the following symmetric positive definite matrix:

$$
S=\left[\begin{array}{cc}
1 & -1 \\
-1 & 2
\end{array}\right]
$$

The state variables of the system (41) are estimated using the following sliding-mode adaptive observer:

$$
\begin{aligned}
& \dot{\hat{x}}_{1}=\hat{x}_{2}-2 \lambda \tilde{e}_{1}-2 \lambda l \arctan \left(k_{o} \tilde{e}_{1}\right) \\
& \dot{\hat{x}}_{2}=\theta^{T} \psi(\underline{\hat{\eta}})-\lambda^{2} \tilde{e}_{1}-\lambda^{2} l \arctan \left(k_{o} \tilde{e}_{1}\right)
\end{aligned}
$$

where $\hat{\eta}=\left[\hat{x}_{1}, \hat{x}_{2}, u\right]^{T}$ and the design parameters are specified as follows, $\gamma_{1}=8, \gamma_{2}=120, \sigma=0.3, k_{0}=1000$ and $\lambda=2$. Note that a small value for $\lambda$ is chosen so as to emphasize the contribution of the fuzzy system for the observation process.

The initial conditions are chosen as, $\hat{x}^{T}(0)=[1,4], x^{T}(0)=[0.5,0], \theta_{i}(0)=0$ (i.e. no a priori information on the fuzzy parameters) and the control input $u$ is a square wave having amplitude \pm 1 with a period of $2 s$ [25].

The membership functions for $\hat{\eta}_{j}$, with $j=1,2,3$, are predefined as follows:

$$
\begin{aligned}
& A_{j}^{1}\left(\hat{\eta}_{j}\right)=\frac{1}{1+\exp \left(18.38\left(\hat{\eta}_{j}+0.75\right)\right)}, \quad A_{j}^{2}\left(\hat{\eta}_{j}\right)=\exp \left(-\frac{\left(\hat{\eta}_{j}+0.5\right)^{2}}{0.09}\right), \quad A_{j}^{3}\left(\hat{\eta}_{j}\right)=\exp \left(-\frac{\hat{\eta}_{j}^{2}}{0.09}\right), \\
& A_{j}^{4}\left(\hat{\eta}_{j}\right)=\exp \left(-\frac{\left(\hat{\eta}_{j}-0.5\right)^{2}}{0.09}\right), \quad A_{j}^{5}\left(\hat{\eta}_{j}\right)=\frac{1}{1+\exp \left(-18.38\left(\hat{\eta}_{j}-0.75\right)\right)}
\end{aligned}
$$

The simulation results of the smooth sliding mode adaptive observer (42) are shown in Fig. 4. The actual and estimated states are shown in Fig. $4 a$ and b, while Fig. $4 \mathrm{c}$ shows the estimated nonlinear function $\hat{f}(\hat{\eta}, \theta)=\theta^{T} \psi(\hat{\eta})$ with the actual nonlinear 


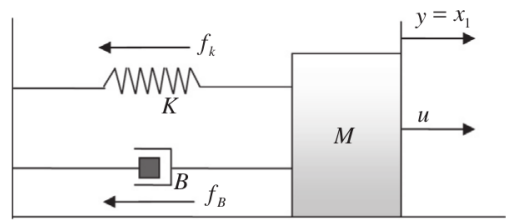

Fig. 3. A mass-spring-damper system.
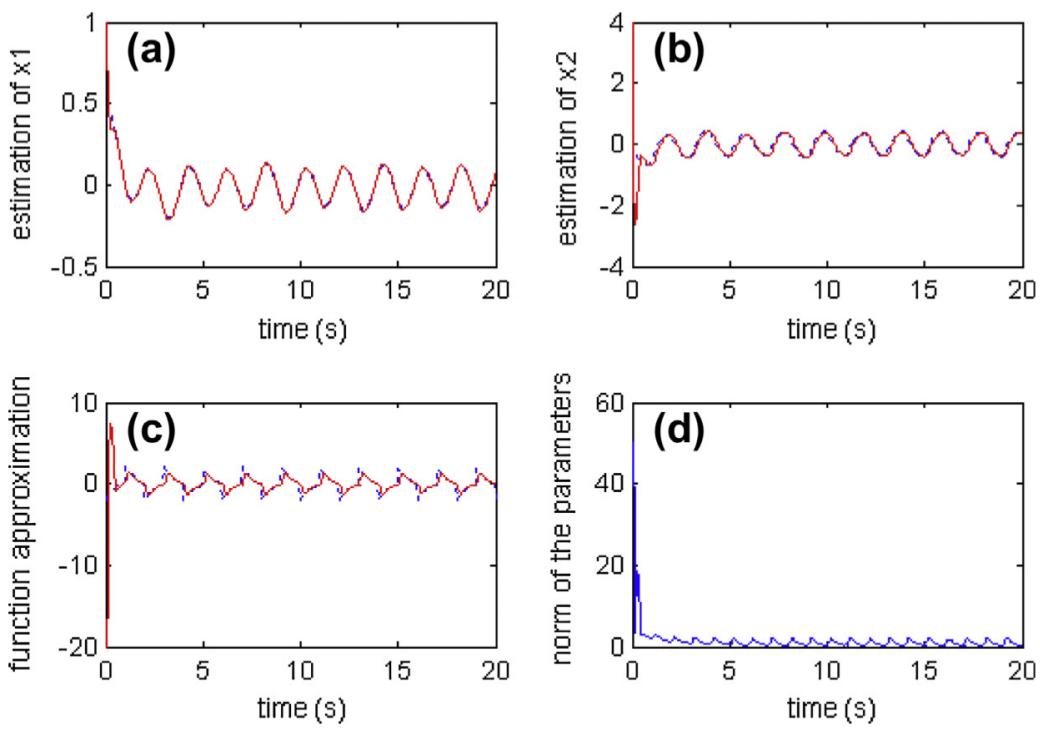

Fig. 4. Simulation results: (a) state $x_{1}$ (dotted line) and its estimate $\hat{x}_{1}$ (solid line). (b) State $x_{2}$ (dotted line) and its estimate $\hat{x}_{2}$ (solid line). (c) The estimated function $\theta^{T} \psi(\underline{\hat{\eta}})$ (solid line) and the actual function $f(\underline{\underline{x}}, u)$ (dotted line). (d) Norm of adaptive parameters $\|\theta\|$.

function $f(\underline{x}, u)$. It is worth noting that $f(\underline{x}, u)$ is only used for simulation purposes, since it is not known by the observer. The norm of the fuzzy parameters is shown in Fig. $4 \mathrm{~d}$.

Fig. $4 \mathrm{a}$ and $\mathrm{b}$ clearly show that the observation error is actually vanishing according to the observer dynamics. The parameter adaptation alertness is particularly emphasized in Fig. $4 \mathrm{c}$ which shows the estimated function $\hat{f}(\underline{\hat{\eta}}, \theta)$ as well as the actual function $f(\underline{x}, u)$.

Example 2. The model of half-car suspension is shown in Fig. 5 [19]. The half-vehicle suspension model is represented by a nonlinear four degree-of-freedom system. It consists in a single sprung mass (car body) connected to two unsprung masses (front and rear wheels) at each corner. The sprung mass is free to heave and pitch, while the unsprung masses are free to bounce vertically with respect to the sprung mass. The suspensions between the sprung mass and unsprung masses are modelled as linear viscous dampers and spring elements, while the tires are modelled as simple linear springs without damping. Applying a force-balance analysis, the equations of motion are given by

$$
\begin{aligned}
\ddot{z}= & \frac{1}{m_{s}}\left[-\left(B_{f}+B_{r}\right) \dot{z}+\left(a B_{f}-b B_{r}\right) \dot{\theta} \cos \theta-k_{f}\left(z_{s f}-z_{u f}\right)-k_{r}\left(z_{s r}-z_{u r}\right)+B_{f} \dot{z}_{u f}+B_{r} \dot{z}_{u r}+f_{f}+f_{r}\right] \\
\ddot{\theta}= & \frac{1}{J_{y}}\left[-\left(a^{2} B_{f}+b^{2} B_{r}\right) \dot{\theta} \cos ^{2} \theta+\left(a B_{f}-b B_{r}\right) \dot{z} \cos \theta+a k_{f}\left(z_{s f}-z_{u f}\right) \cos \theta-b k_{r}\left(z_{s r}-z_{u r}\right) \cos \theta\right. \\
& \left.-\left(a B_{f} \dot{z}_{u f}-b B_{r} \dot{z}_{u r}\right) \cos \theta-a f_{f} \cos \theta+b f_{r} \cos \theta\right] \\
\ddot{z}_{u f}= & \frac{1}{m_{u f}}\left[B_{f}\left(\dot{z}-a \dot{\theta} \cos \theta-\dot{z}_{u f}\right)+\left(k_{f}+K_{t f}\right)\left(z_{s f}-z_{u f}\right)-K_{t f} z+a K_{t f} \sin \theta+K_{t f} z_{r f}-f_{f}\right] \\
\ddot{z}_{u r}= & \frac{1}{m_{u r}}\left[B_{r}\left(\dot{z}+b \dot{\theta} \cos \theta-\dot{z}_{u r}\right)+\left(k_{r}+K_{t r}\right)\left(z_{s r}-z_{u r}\right)-K_{t r} z-b K_{t r} \sin \theta+K_{t r} z_{r r}-f_{r}\right]
\end{aligned}
$$

where $z_{s f}=z-a \sin \theta$ is the front body displacement, $z_{s r}=z+b \sin \theta$ is the rear body displacement and $J_{y}=m_{s} r_{y}^{2}$ is the centroidal moment of inertia. Different notations of this half-car model are given in Table 3. 


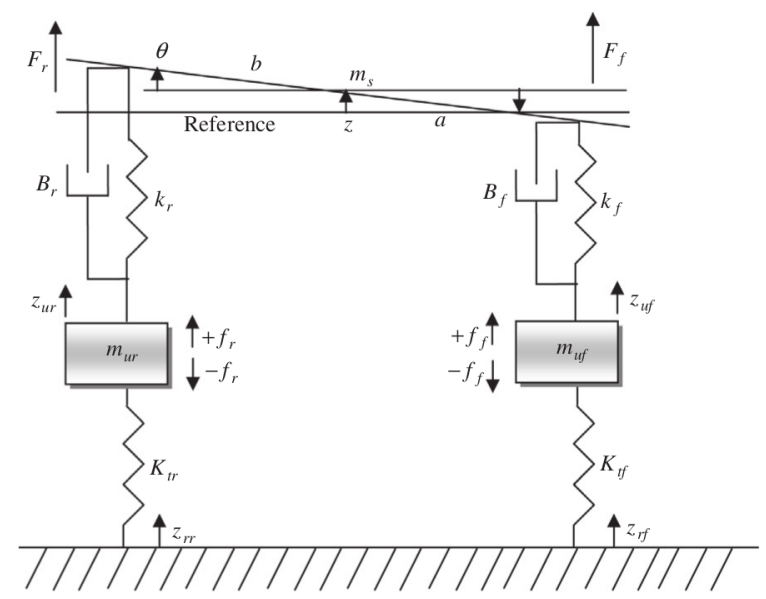

Fig. 5. Half-car suspension model.

Table 3

Parameters and variables of the half-car active suspension model.

\begin{tabular}{|c|c|c|c|}
\hline$f, r$ & Subscripts of front and rear & $z, \dot{z}$ & Heave (ride height of sprung mass) and payload velocity \\
\hline$K_{t r}, K_{t f}$ & Tire spring coefficients & $\theta, \dot{\theta}$ & Pitch angle and pitch velocity \\
\hline$k_{r}, k_{f}$ & Spring coefficients & $z_{u f}, \dot{z}_{u f}$ & Displacement and velocity of the front wheel \\
\hline$B_{r}, B_{f}$ & Damping coefficients & $z_{u r}, \dot{z}_{u r}$ & Displacement and velocity of the rear wheel \\
\hline$m_{u r}, m_{u f}$ & Unsprung masses & $z_{s f}, \dot{z}_{s f}$ & Displacement and velocity of the front car body \\
\hline$a, b$ & Distance of axles from centroid & $z_{S r}, \dot{z}_{S r}$ & Displacement and velocity of the rear car body \\
\hline$m_{s}, J_{y}$ & Mass of body and Inertia moment & $\begin{array}{l}z_{r f}, z_{r r} \\
f_{f}, f_{r}\end{array}$ & $\begin{array}{l}\text { The front and rear terrain height disturbances } \\
\text { The front and rear force inputs }\end{array}$ \\
\hline
\end{tabular}

To design the observer, let us define the state vector as follows:

$$
\underline{x}=\left[x_{1}, x_{2}, x_{3}, x_{4}, x_{5}, x_{6}, x_{7}, x_{8}\right]^{T}=\left[z, \dot{z}, \theta, \dot{\theta}, z_{u f}, \dot{z}_{u f}, z_{u r}, \dot{z}_{u r}\right]^{T}
$$

This results in the system state equation given below

$$
\begin{aligned}
\dot{x}_{1}= & x_{2} \\
\dot{x}_{2}= & \frac{1}{m_{s}}\left[-\left(B_{f}+B_{r}\right) x_{2}+\left(a B_{f}-b B_{r}\right) x_{4} \cos x_{3}-k_{f}\left(x_{1}-a \sin x_{3}-x_{5}\right)-k_{r}\left(x_{1}+b \sin x_{3}-x_{7}\right)+B_{f} x_{6}+B_{r} x_{8}+u_{1}+u_{2}\right] \\
\dot{x}_{3}= & x_{4} \\
\dot{x}_{4}= & \frac{1}{J_{y}}\left[-\left(a^{2} B_{f}+b^{2} B_{r}\right) x_{4} \cos ^{2} x_{3}+\left(a B_{f}-b B_{r}\right) x_{2} \cos x_{3}+a k_{f}\left(x_{1}-a \sin x_{3}-x_{5}\right) \cos x_{3}-b k_{r}\left(x_{1}+b \sin x_{3}-x_{7}\right) \cos x_{3}\right. \\
& \left.-\left(a B_{f} x_{6}-b B_{r} x_{8}\right) \cos x_{3}-a u_{1} \cos x_{3}+b u_{2} \cos x_{3}\right] \\
\dot{x}_{5}= & x_{6} \\
\dot{x}_{6}= & \frac{1}{m_{u f}}\left[B_{f}\left(x_{2}-a x_{4} \cos x_{3}-x_{6}\right)+\left(k_{f}+K_{t f}\right)\left(x_{1}-a \sin x_{3}-x_{5}\right)-K_{t f} x_{1}+a K_{t f} \sin x_{3}+K_{t f} z_{r f}-u_{1}\right] \\
\dot{x}_{7}= & x_{8} \\
\dot{x}_{8}= & \frac{1}{m_{u r}}\left[B_{r}\left(x_{2}+b x_{4} \cos x_{3}-x_{8}\right)+\left(k_{r}+K_{t r}\right)\left(x_{1}+b \sin x_{3}-x_{7}\right)-K_{t r} x_{1}-b K_{t r} \sin x_{3}+K_{t r} z_{t r}-u_{2}\right]
\end{aligned}
$$

where $u_{1}=f_{f}$ and $u_{1}=f_{r}$.

Dynamic system (43) can be finally rewritten in the following compact form:

$$
\dot{x}_{1}=x_{2}
$$



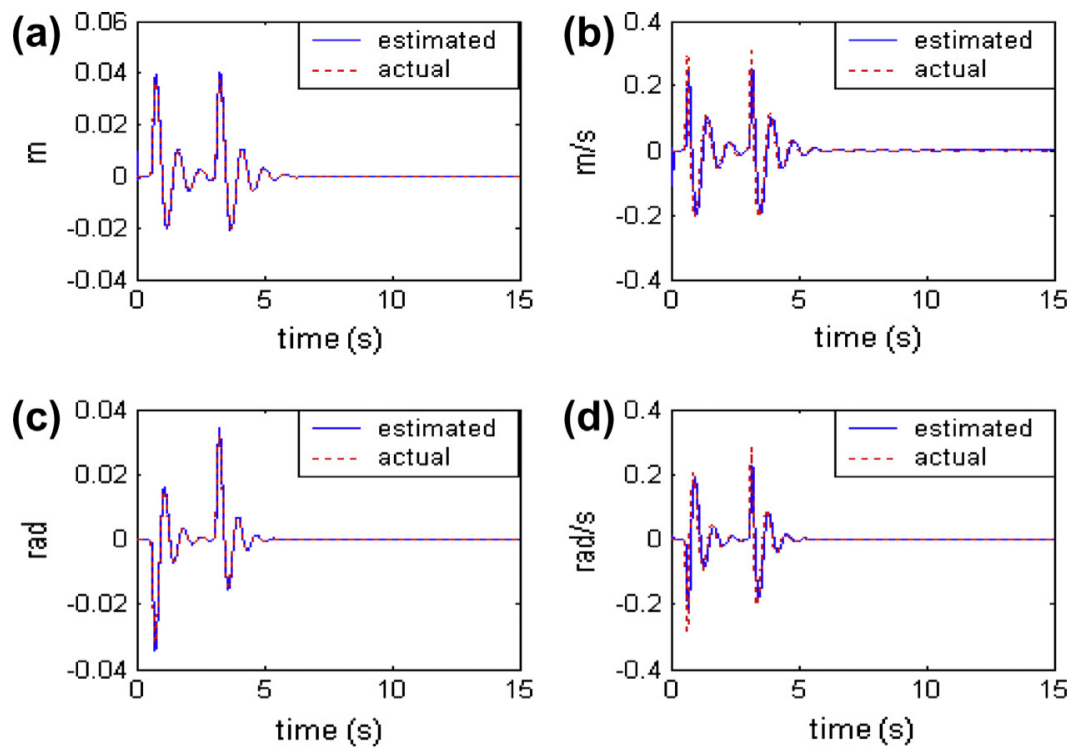

Fig. 6. Simulation results for the passive suspension. (a) Heave (ride height of sprung mass). (b) Payload velocity. (c) Pitch angle. (d) Pitch velocity.

$$
\begin{aligned}
& \dot{x}_{2}=f_{1}\left(\underline{x}, u_{1}, u_{2}\right) \\
& \dot{x}_{3}=x_{4} \\
& \dot{x}_{4}=f_{2}\left(\underline{x}, u_{1}, u_{2}\right) \\
& \dot{x}_{5}=x_{6} \\
& \dot{x}_{6}=f_{3}\left(\underline{x}, u_{1}, u_{2}\right)+d_{1} \\
& \dot{x}_{7}=x_{8} \\
& \dot{x}_{8}=f_{4}\left(\underline{x}, u_{1}, u_{2}\right)+d_{2} \\
& y=\left[x_{1}, x_{3}, x_{5}, x_{7}\right]^{T}
\end{aligned}
$$

where $d_{1}=K_{t f} z_{r f} / m_{u f}, d_{2}=K_{t r} z_{r r} / m_{u r}$ and $y$ is the output vector which is assumed to be available for measurement. The functions $f_{i}\left(\underline{x}, u_{1}, u_{2}\right)$ gather all subsystems nonlinearities. This model will be used later in the observer design.

For simulation purposes, we will use the following control law proposed in [19] for the half-car active suspension:

$$
\begin{aligned}
u_{1}= & m_{s}\left[\kappa\left(k_{t f}+K_{t r}\right) x_{2}-\kappa K_{t f}\left(x_{2}-a x_{4} \cos x_{3}-x_{6}\right)+\left(c_{1}^{2}-1\right) z_{1}\right]+\left(B_{f}+B_{r}\right) x_{2}+k_{f}\left(x_{1}-a \sin x_{3}-x_{5}\right)-B_{f} x_{6} \\
u_{2}= & -m_{s}\left[\kappa\left(a k_{t f}-b K_{t r}\right) x_{4} \cos x_{3}+\kappa K_{t r}\left(x_{2}+b x_{4} \cos x_{3}-x_{8}\right)+\left(c_{1}+c_{2}\right) z_{2}\right]-\left(a B_{f}-b B_{r}\right) x_{4} \cos x_{3}+k_{r}\left(x_{1}+b\right. \\
& \left.\times \sin x_{3}-x_{7}\right)-B_{r} x_{8}
\end{aligned}
$$

with

$$
\begin{aligned}
& z_{1}=x_{1}+\kappa\left(m_{s} x_{2}+m_{u f} x_{6}+m_{u r} x_{8}\right) \\
& z_{2}=x_{2}-\kappa\left[\left(K_{t f}+K_{t r}\right) x_{1}-\left(a K_{t f}-b K_{t r}\right) \sin x_{3}-K_{t f}\left(x_{1}-a \sin x_{3}-x_{5}\right)\right. \\
& \left.-K_{t r}\left(x_{1}+b \sin x_{3}-x_{7}\right)\right]-c_{1} z_{1}
\end{aligned}
$$

where $c_{1}, c_{2}$ and $\kappa$ are positive design constants. Even though the control system design has been carried out for the half-car suspension model (43), the observer design has been performed without assuming any knowledge of the system as well as the control law (46). The considered control system is mainly for simulation purposes.

It is clear that the system (45) can be considered as a set of the subsystems having the form (2). Then, one can design the following coupled fuzzy adaptive observers to estimate the state vector $\underline{x}$ : 


$$
\begin{aligned}
& \hat{x}_{1}=\hat{x}_{2}-2 \lambda \tilde{e}_{1} \\
& \dot{\hat{x}}_{2}=\theta_{1}^{T} \psi_{1}\left(\hat{\hat{\eta}}_{1}\right)-\lambda^{2} \tilde{e}_{1} \\
& \dot{\hat{x}}_{3}=\hat{x}_{4}-2 \lambda \tilde{e}_{3} \\
& \dot{\hat{x}}_{4}=\theta_{2}^{T} \psi_{2}\left(\hat{\hat{\eta}}_{2}\right)-\lambda^{2} \tilde{e}_{3} \\
& \dot{\hat{x}}_{5}=\hat{x}_{6}-2 \lambda \tilde{e}_{5} \\
& \dot{\hat{x}}_{6}=\theta_{3}^{T} \psi_{3}\left(\underline{\hat{\eta}}_{3}\right)-\lambda^{2} \tilde{e}_{5} \\
& \dot{\hat{x}}_{7}=\hat{x}_{8}-2 \lambda \tilde{e}_{7} \\
& \dot{\hat{x}}_{8}=\theta_{4}^{T} \psi_{4}\left(\hat{\eta}_{4}\right)-\lambda^{2} \tilde{e}_{7}
\end{aligned}
$$

For simplicity purposes, the input vectors of the adaptive fuzzy systems are selected here as follows: $\hat{\eta}_{1}=\underline{\hat{\eta}}_{2}=\hat{\hat{\eta}}_{3}=\underline{\hat{\eta}}_{4}=\underline{\hat{x}}$ where $\underline{\hat{x}}$ is the estimate of the state vector $\underline{x}$. Notice that the functions $f_{i}\left(\underline{x}, u_{1}, u_{2}\right)$ depend implicitly of $\underline{x}$ since the control law is a function of state $\underline{x}$.

The parameters of the half-car model selected for this study are [19]:

$$
\begin{aligned}
& m_{s}=575 \mathrm{~kg}, \quad m_{u f}=m_{u r}=60 \mathrm{~kg}, J_{y}=769 \mathrm{~kg} / \mathrm{m}^{2}, \quad a=1.38 \mathrm{~m}, \quad b=1.36 \mathrm{~m} \\
& k_{f}=k_{r}=16,812 \mathrm{~N} / \mathrm{m}, \quad B_{f}=B_{r}=1000 \mathrm{~N} / \mathrm{m} / \mathrm{s}, \quad K_{t f}=K_{t r}=190,000 \mathrm{~N} / \mathrm{m}
\end{aligned}
$$

and the road disturbances employed on the front and rear wheels are:

$$
\begin{array}{ll}
z_{r f}(t)=\mu_{r}(1-\cos (8 \pi t)), & \text { for } 0.5 \leqslant t \leqslant 0.75 \\
z_{r r}(t)=\mu_{r}(1-\cos (8 \pi t)), & \text { for } 3 \leqslant t \leqslant 3.25
\end{array}
$$

where $\mu_{r}$ is the amplitude (meters).

The design constants for controller are selected as in [19]:

$$
c_{1}=c_{2}=1, \quad \kappa=0.000003
$$

For all observers of the subsystems, the design parameters are specified as follows:

$$
\gamma_{1}=50, \quad \gamma_{2}=50, \quad \sigma=0.1, \quad \text { and } \lambda=15
$$
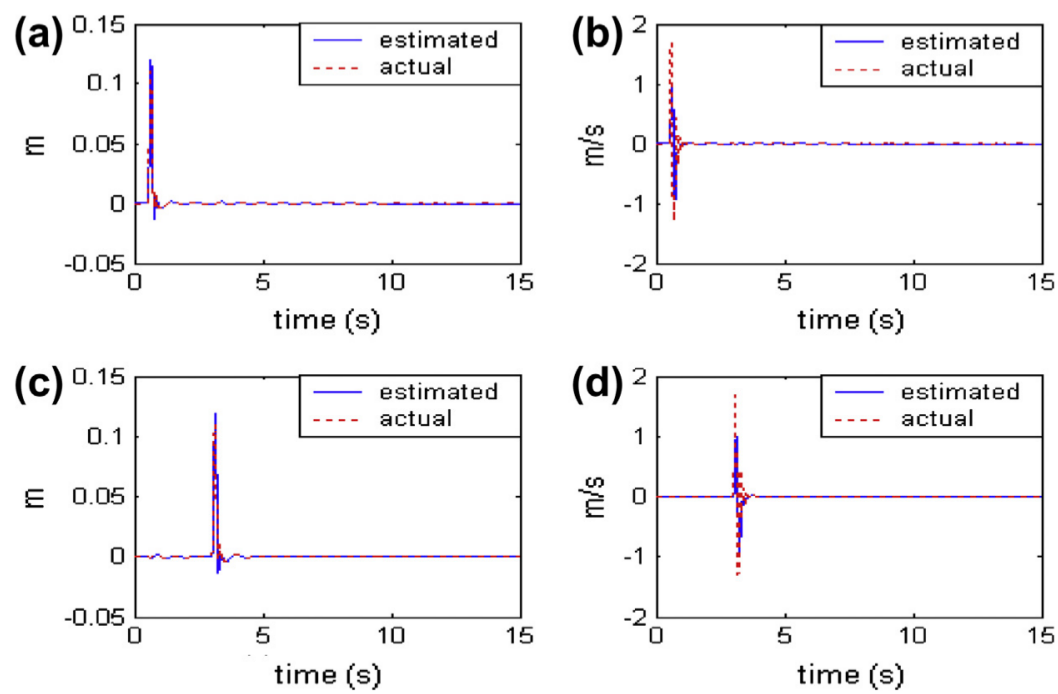

Fig. 7. Simulation results for the passive suspension. (a) Displacement of the front wheel. (b) Velocity of the front wheel. (c) Displacement of the rear wheel. (d) Velocity of the rear wheel. 

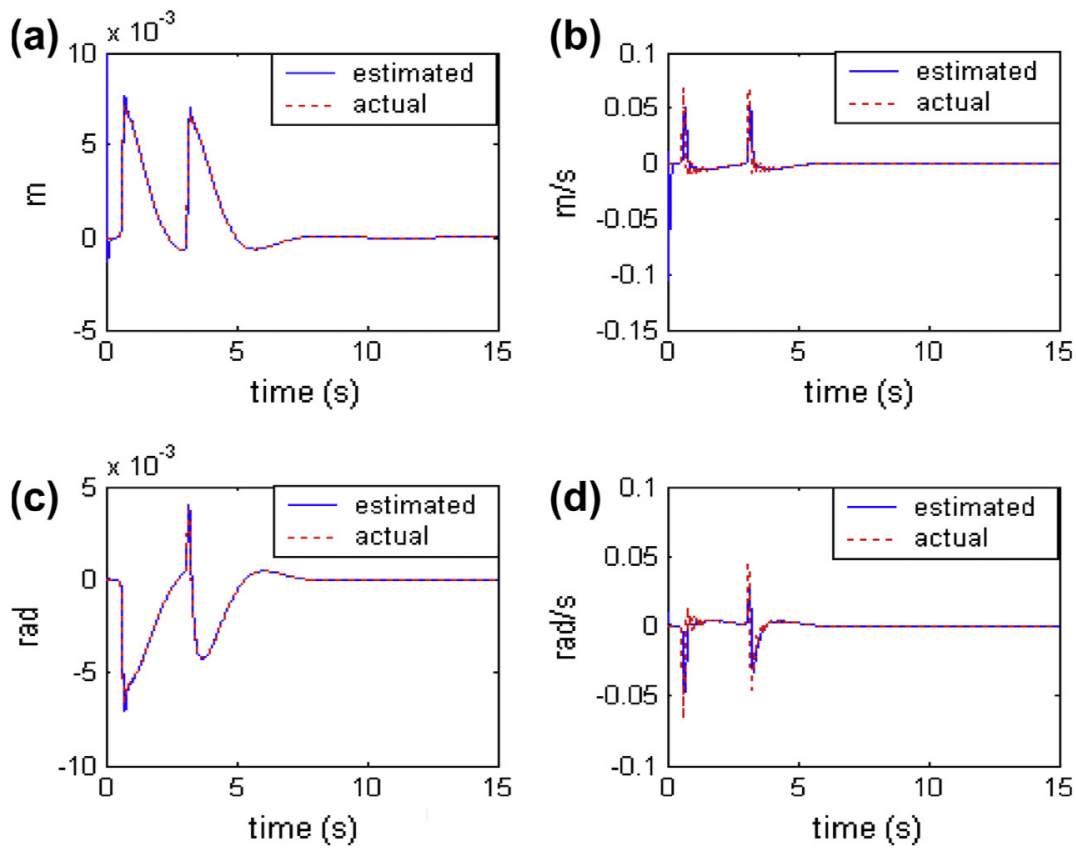

Fig. 8. Simulation results for the active suspension (without noise). (a) Heave (ride height of sprung mass). (b) Payload velocity. (c) Pitch angle. (d) Pitch velocity.
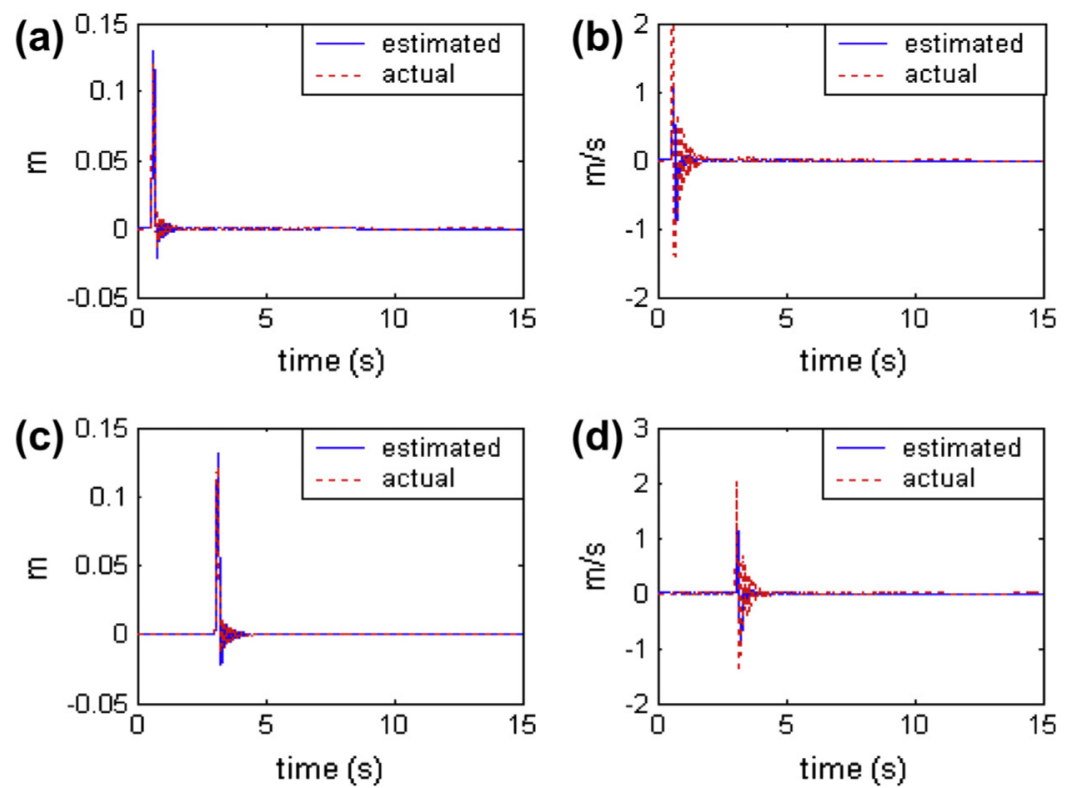

Fig. 9. Simulation results for the active suspension (without noise). (a) Displacement of the front wheel. (b) Velocity of the front wheel. (c) Displacement of the rear wheel. (d) Velocity of the rear wheel.

For each variable of the entries (of the fuzzy systems), one defines three triangular membership functions uniformly distributed on the following intervals: $[-0.04,0.04]$ for $\hat{x}_{1}, \hat{x}_{3},[-0.2,0.12]$ for $\hat{x}_{5}, \hat{x}_{7},[-0.4,0.4]$ for $\hat{x}_{2}, \hat{x}_{4}$ and $[-1.5,1.5]$ for $\hat{x}_{6}, \hat{x}_{8}$.

The initial values of the fuzzy parameters are chosen as, $\theta_{i}(0)=\underline{0}$ (i.e. no a priori information on the fuzzy parameters).

Three different simulation cases are considered to demonstrate the performances of the proposed adaptive fuzzy observer.

(a) Case 1: The fuzzy adaptive observer (47) is used to estimate the states of the standard passive suspension. The simulation results are shown in Figs. 6 and 7. Notice that the estimated values of the state variables are close to the actual ones. 

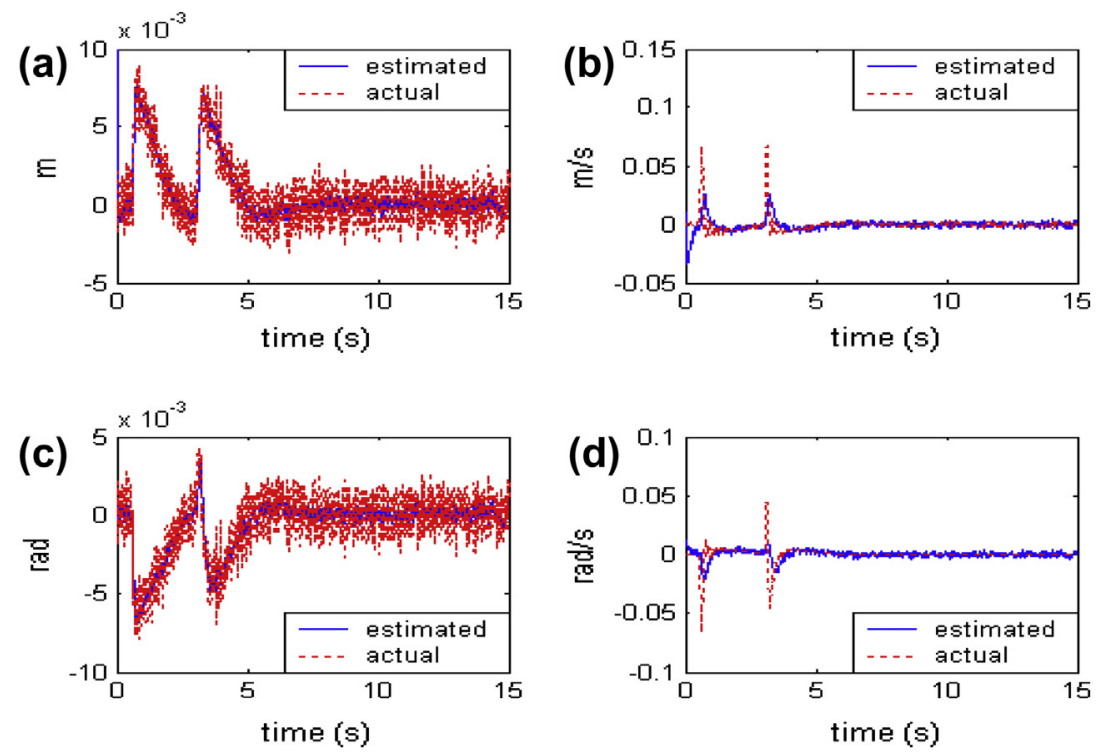

Fig. 10. Simulation results for the active suspension (with noise). (a) Heave (ride height of sprung mass). (b) Payload velocity. (c) Pitch angle. (d) Pitch velocity.
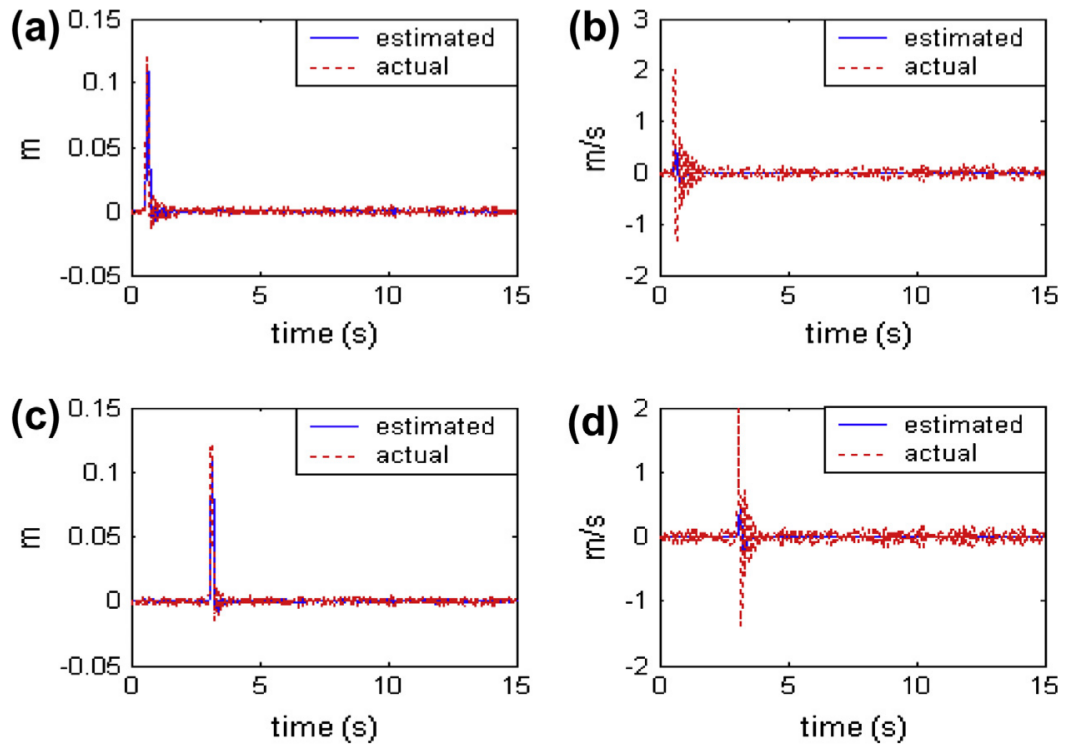

Fig. 11. Simulation results for the active suspension (with noise). (a) Displacement of the front wheel. (b) Velocity of the front wheel. (c) Displacement of the rear wheel. (d) Velocity of the rear wheel.

(b) Case 2: The observer (47) is used to estimate the states of the active suspension. The simulation results shown in Figs. 8 and 9 emphasize the estimation performances.

(c) Case 3: To simulate practical situations, the output measurements have been corrupted by an additive noise. Figs. 10 and 11 show the simulation results obtained on the active suspension in the presence of noise measurements. From these results, one can notice that the considered fuzzy adaptive observer is not quite sensitive to noise measurements.

\section{Conclusion}

In this work, a unified adaptive fuzzy observer design framework for uncertain nonlinear systems has been proposed. The adaptive fuzzy system is used to approximate an uncertain nonlinear function. The proposed observer presents some design flexibility and can be easily adaptable into high gain based observers, namely the usual high gain observers and the sliding modes observers together with their practically oriented versions, and a PI parameter adaptation law for updating the fuzzy 
parameters. The usual SPR condition as well as the error filtering are no longer required. The approximation error and external disturbances can be appropriately reduced in the proposed observation design framework thanks to the proportional action involved in this PI adaptation law. A Lyapunov based approach has been adopted to show that the involved observation and parameter errors are ultimately uniformly bounded. The effectiveness of the proposed adaptive fuzzy observers has been illustrated via simulation results involving a mass-spring-damper system and a half-car active suspension system.

\section{References}

[1] J.P. Barbot, T. Boukhobza, M. Djemai, Sliding mode observer for triangular input form, in: Proc. 35th IEEE Conf. on Decision and Control, 1996, pp. 1489-1490.

[2] G. Bastin, M.R. Gevers, Stable adaptive observers for nonlinear time-varying systems, IEEE Trans. Autom. Control 33 (1988) 650-658.

[3] Y.J. Cao, A nonlinear adaptive approach to controlling chaotic oscillators, Phys. Lett. A 270 (2000) 171-176.

[4] J.L. Castro, Fuzzy logic controllers are universal approximators, IEEE Trans. Syst. Man Cybernet. 25 (4) (1995) 629-635.

[5] Y.M. Cho, R. Rajamani, A systematic approach to adaptive observer synthesis for nonlinear systems, IEEE Trans. Autom. Control 42 (1997) 534-537.

[6] J.Y. Choi, J. Farrell, Adaptive observer for a class of nonlinear systems using neural networks, in: Proc. of the 1999 IEEE International Symposium on Intelligent Control/Intelligent Systems and Semiotics, Cambridge, MA, 1999, pp. 114-119.

[7] M. Farza, M. M'saad, Observer design for a class of MIMO nonlinear systems, Automatica 40 (2004) 135-143.

[8] M. Farza, M. Msaad, M. Sekher, A set of observers for a class of nonlinear systems, in: Proc. of the IFAC Word Congress, Prague, Czech Republic, 2005.

[9] A. Filipescu, L. Dugard, J.-M. Dion, Adaptive gain sliding mode observer based sliding controller for uncertain parameters nonlinear systems: application to flexible joint robots, in: Proc. 42nd IEEE Conf. on Decision and Control, 2003, pp. 3537-3542.

[10] J.P. Gauthier, H. Hammouri, S. Othman, A simple observer for nonlinear systems, applications to bioreactors, IEEE Trans. Autom. Control 37 (6) (1992) $875-880$.

[11] K. Hornik, Multilayer feedforward networks are universal approximators, Neural Netw. 2 (1989) $359-366$.

[12] C. Hua, F. Li, X. Guan, Observer-based adaptive control for uncertain time-delay systems, Inform. Sci. 176 (2) (2006) $201-214$.

[13] P.A. Ioannou, P.V. Kokotovic, Instability analysis and improvement of robustness of adaptive control, Automatica 20 (5) (1984) $583-594$.

[14] P.A. Ioannou, J. Sun, Robust Adaptive Control, Prentice-Hall, Englewood Cliffs, NJ, 1996.

[15] L. Jiang, Q.H. Wu, Nonlinear adaptive control via sliding-mode state and perturbation observer, IEE Proc. Control Theory Appl. 149 (4) (2002) $269-277$.

[16] L. Jiang, Q.H. Wu, C. Zhang, X.X. Zhou, Observer-based nonlinear control of synchronous generators with perturbation estimation, Electr. Power Energy Syst. 23 (2001) 359-367.

[17] Y.H. Kim, F.L. Lewis, C.T. Abdallah, A dynamic recurrent neural-network-based adaptive observer for a class of nonlinear systems, Automatica 33 (8) (1997) 1539-1543.

[18] Y.G. Leu, T.T. Lee, W.Y. Wang, Observer-based adaptive fuzzy-neural control for unknown nonlinear dynamical systems, IEEE Trans. Syst. Man Cybernet. - Part B: Cybernet. 29 (1999) 583-591.

[19] J.S. Lin, C.J. Huang, Nonlinear backstepping control design of half-car active suspension systems, Int. J. Veh. Des. 33 (4) (2003) $332-350$.

[20] C.-K. Lin, Robust adaptive critic control of nonlinear systems using fuzzy basis function networks: an LMI approach, Inform. Sci. 177 (22) (2007) 4934-4946.

[21] Y.-J. Liu, W. Wang, Adaptive fuzzy control for a class of uncertain nonaffine nonlinear systems, Inform. Sci. 177 (18) (2007) $3901-3917$.

[22] R. Morino, Adaptive observers for single output nonlinear systems, IEEE Trans. Autom. Control 35 (1990) 1054-1058.

[23] R. Morino, P. Tomie, Global adaptive observers for nonlinear systems via filtered transformations, IEEE Trans. Autom. Control 37 (1992) $1239-1245$.

[24] R. Morino, P. Tomie, Adaptive observers with arbitrary exponential rate of convergence for nonlinear systems via filtered transformations, IEEE Trans. Autom. Control 40 (1995) 1300-1304.

[25] J.H. Park, G.-T. Park, Adaptive fuzzy observer with minimal dynamic order for uncertain nonlinear systems, IEE Proc. Control Theory Appl. 150 (2) (2003) 189-197.

[26] J.H. Park, G.T. Park, S.H. Kim, C.J. Moon, Output feedback control of uncertain nonlinear systems using a self-structuring adaptive fuzzy observer, Fuzzy Sets Syst. 151 (2005) 21-42.

[27] R. Rajamani, K. Hedrick, Adaptive observer for active automotive suspensions: theory and experiment, IEEE Trans. Control Syst. Technol. 3 (1995) $86-93$.

[28] S. Seshagiri, H.K. Khalil, Output feedback control of nonlinear systems using RBF neural networks, IEEE Trans. Neural Netw. 11 (1) (2000) 69-79.

[29] C.S. Ting, An observer-based approach to controlling time-delay chaotic systems via Takagi-Sugeno fuzzy model, Inform. Sci. 177 (20) (2007) 4314-4328.

[30] S. Tong, Y. Li, P. Shi, Fuzzy adaptive backstepping robust control for SISO nonlinear system with dynamic uncertainties, Inform. Sci. 179 (2009) $1319-1332$.

[31] S. Tong, Y. Li, Fuzzy adaptive robust backstepping stabilization for SISO nonlinear systems with unknown virtual control direction, Inform. Sci. 180 (2010) 4619-4640.

[32] S. Tong, X. He, Y. Li, Direct adaptive fuzzy backstepping robust control for single input and single output uncertain nonlinear systems using small-gain approach, Inform. Sci. 180 (2010) 1738-1758.

[33] A.B. Trunov, M.M. Polycarpou, Automated fault diagnosis in nonlinear multivariable systems using a learning methodology, IEEE Trans. Neural Netw. 10 (2000) 91-101.

[34] J.A.R. Vargas, E.M. Hemerly, Adaptive observers for unknown general nonlinear systems, IEEE Trans. Syst. Man Cybernet. - Part B: Cybernet. 31 (5) (2001) 683-690.

[35] M. Wang, B. Chen, K. Liu, X. Liu, S. Zhang, Adaptive fuzzy tracking control of nonlinear time-delay systems with unknown virtual control coefficients, Inform. Sci. 178 (2008) 4326-4340.

[36] L.X. Wang, Adaptive Fuzzy Systems and Control: Design and Stability Analysis, Prentice-Hall, Englewood Cliffs, NJ, 1994.

[37] L.X. Wang, J.M. Mendel, Fuzzy basis functions, universal approximation, and orthogonal least square learning, IEEE Trans. Neural Netw. 3 (5) (1992) $807-814$.

[38] Y. Wang, T. Chai, Y. Zhang, State observer-based adaptive fuzzy output-feedback control for a class of uncertain nonlinear systems, Inform. Sci. 180 (2010) 5029-5040.

[39] Y. Xiong, M. Saif, Sliding mode observer for nonlinear uncertain systems, IEEE Trans. Autom. Control 46 (12) (2001) $2012-2017$.

[40] A. Xu, Q. Zhang, Nonlinear system fault diagnostic based on adaptive estimation, Automatica 40 (2004) 1181-1193.

[41] Y. Yang, C. Zhou, Robust adaptive fuzzy tracking control for a class of perturbed strict-feedback nonlinear systems via small-gain approach, Inform. Sci. $170(2-4)(2005) 211-234$.

[42] S.J. Yoo, J.B. Park, Decentralized adaptive output-feedback control for a class of nonlinear large-scale systems with unknown time-varying delayed interactions, Inform. Sci. 186 (2012) 222-238.

[43] W. Yu, M.A. Moreno-Armendariz, F.O. Rodriguez, Stable adaptive compensation with fuzzy CMAC for an overhead crane, Inform. Sci. 181 (2011) 4895-4907.

[44] R. Zhu, T. Chai, C. Shao, Robust nonlinear adaptive observer design using dynamical recurrent neural networks, in: Proc. ACC, Albuquerque, NM, June 1997, pp. 1096-1100. 